\title{
Influence of Nonalcoholic Fatty Liver Disease on Locally Advanced Breast Cancer: A Retrospective Cohort Study
}

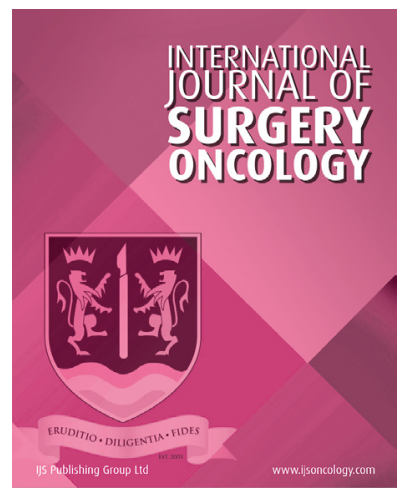

\author{
TOMOE TAJI (1) \\ YUKI KATAOKA (D) \\ HIROFUMI SUWA
}

AI YAMAGUCHI (D)

*Author affiliations can be found in the back matter of this article
KAZUNA KAWABATA

MARINA SHIMIZU

MAKOTO UMEDA
CORRESPONDING AUTHOR:

\section{Tomoe Taji}

Department of Breast Surgery, Hyogo Prefectural Amagasaki General Medical Center, 2-17-77, Higashinaniwa-cho, Amagasaki, Hyogo 660-8550, Japan

tajit.mutsu@gmail.com

\section{KEYWORDS:}

Nonalcoholic fatty liver disease; locally advanced breast cancer; prognosis

TO CITE THIS ARTICLE: Taji T, Kataoka Y, Suwa H, Yamaguchi A, Kawabata K, Shimizu M, Umeda M. Influence of Nonalcoholic Fatty Liver Disease on Locally Advanced Breast Cancer: A Retrospective Cohort Study. International Journal of Surgery: Oncology. 2021; 6(1), 18-24. DOI: https://doi. org/10.29337/ijsonco.63

\section{Highlights:}

- This single-center, retrospective, cohort study investigated the association between nonalcoholic fatty liver disease (NAFLD) and the prognosis and morbidity of patients with locally advanced breast cancer ( $L A B C$ ).

- We found that mild NAFLD was not significantly associated with RFS and neither cardiovascular disease nor other cancers occurred.

- The results of the current study imply that mild NAFLD based on the criteria given by Iwasaki et al. is not a prognostic factor for $L A B C$. 


\section{INTRODUCTION}

Nonalcoholic fatty liver disease (NAFLD) is a common comorbidity in patients with breast cancer and is associated with poor prognosis. Patients with breast cancer have a higher rate of NAFLD than the general population does [1, 2]. Two retrospective cohort studies showed that patients with breast cancer with NAFLD were at a higher risk of recurrence. One study was from Korea and showed that recurrence-free survival (RFS) after radical surgery was significantly longer in the control group than in the NAFLD group, and NAFLD was a risk factor for recurrence on multivariate analysis [2]. The other study was from China and demonstrated that the patients diagnosed with NAFLD during follow-up had significantly shorter RFS than those in the control group did. The study included patients with estrogen receptorpositive, human epidermal growth factor receptor 2-negative early-stage breast cancer who received adjuvant tamoxifen [3]

Furthermore, people with NAFLD have worse prognosis than those without NAFLD do. Cohort studies showed that more number of liver-related and cardiovascular deaths occurred among patients with NAFLD [4, 5].

The best treatment for patients with locally advanced breast cancer (LABC) with NAFLD is unclear. LABC has a higher rate of distant metastases and local recurrence than early-stage breast cancer does. Standard curative therapy for $L A B C$ includes multidisciplinary treatment: chemotherapy followed by local treatment (surgery and radiation) [6]. Because of the use of anthracyclines, which carry a risk of cardiovascular events [7, 8], and the use of hepatic metabolic anticancer agents, the prognostic impact of NAFLD at the start of treatment is a concern. No previous studies have evaluated the association between NAFLD and LABC. Therefore, we decided to investigate the association between NAFLD and the prognosis and morbidity of patients with $\angle A B C$ in the current retrospective cohort study.

\section{PATIENTS AND METHODS PATIENTS}

We followed the STROBE statement. This study is fully compliant with the STROCSS criteria [9] (Supplementary Table 1).

We retrospectively enrolled patients with stage III breast cancer who were histologically diagnosed with invasive breast cancer and who underwent radical surgery at Hyogo Prefectural Amagasaki General Medical Center between July 2015 and December 2018.

Staging was based on the 8th edition of the UICC TNM classification of malignant tumors [10]. Invasive breast carcinoma included invasive carcinoma of no special type, invasive lobular carcinoma, mucinous carcinoma, and metaplastic carcinoma [11].
We excluded patients who had inflammatory breast cancer, concurrent malignancies, or previous malignant diseases within 5 years. We also excluded patients with positivity for hepatitis B surface antigen or hepatitis $C$ virus antibodies, daily alcohol consumption of $20 \mathrm{~g}$ or more, autoimmune hepatitis, primary biliary cholangitis, and elevated levels of aspartate aminotransferase or alanine transaminase more than three times the upper limit of the normal. We could not evaluate patients who had not undergone pre-treatment computed tomography (CT).

\section{ETHICAL CONSIDERATION}

The study protocol was approved by the Ethics Committee of Hyogo Prefectural Amagasaki General Medical Center (approval number 2-27). Our registration unique identifying number (UIN) is UMIN000043291. The need for patient consent was waived because the patient records were anonymized and de-identified before analysis.

\section{DATA COLLECTION}

The cutoff date for data collection was March 2020. According to the Surveillance, Epidemiology, and End Results (SEER) data, the median survival for stage III is 4.9 years [12]. The overall survival of metastatic breast cancer varies among subtypes and ranges from 13 to 44 months [13]. Therefore, we considered the follow-up period required for at least two years.

Data were collected from the medical records, including the history of hypertension, dyslipidemia, and diabetes mellitus; the smoking status; alcohol consumption status; menopausal status; medications used; pre-treatment blood test data; and body mass index. Data about the pathological findings from biopsies and surgical specimens as well as the treatment options were also obtained.

Breast cancer recurrences were diagnosed by using biopsies or imaging such as $\mathrm{CT}$, positron emission tomography/computed tomography (PET/CT), and magnetic resonance imaging.

\section{EVALUATION OF HEPATIC STEATOSIS}

Hepatic steatosis was evaluated by using the ratio of liverto-spleen attenuation (L/S) on pre-treatment unenhanced $\mathrm{CT}$. Two independent radiologists measured the $\mathrm{CT}$ values of the liver and spleen from 3 regions of interest, and we calculated the mean value. We defined a L/S of $<1.12$ as mild fatty liver and $<0.90$ as severe fatty liver [14]. PET/CT data were used when non-contrast CT data were absent.

\section{DEFINITION OF THE PRIMARY OUTCOMES}

The primary outcome was recurrence-free survival (RFS). RFS was defined as the duration from the date of biopsy to the date of death or distant recurrence, local recurrence, or diagnosis of secondary cancer. Survival confirmation was the last visit to our hospital. If we had not seen the patient for a while, we contacted the general practitioner or patient. 


\section{STATISTICAL ANALYSIS}

We performed descriptive statistics using summary statistics. We used the log-rank test to compare the survival curves between the two groups (patients with NAFLD and those without NAFLD). We used the Cox proportional hazards model to adjust for covariates.

Statistical analysis was performed using JMP version 11 (SAS Institute Inc., USA). A two-sided test was adopted, with $p<0.05$ indicating statistical significance. We conducted complete-case analysis.

\section{RESULTS}

After applying the exclusion criteria, we finally analyzed 44 patients with stage III breast cancer (Figure 1). The median follow-up was 852.5 days (range: 96-1770 days).
Each radiologist diagnosed 10 patients with mild fatty liver (L/S range, radiologist 1: 0.920-1.117, radiologist 2: 0.965-1.113), among whom 6 patients were diagnosed by both radiologists (L/S range, radiologist 1: 0.920-1.114, radiologist 2: 1.022-1.113). We defined these 6 patients as having NAFLD. No patient was diagnosed with severe fatty liver.

We compared the baseline characteristics between patients with NAFLD and those without NAFLD (Table 1).

The median RFS was 713.5 days (range: 96-1770 days). We compared the RFS of the patients with NAFLD and those without NAFLD. On univariate analysis, NAFLD was not significantly associated with RFS (hazard ratio: 1.474, 95\% confidence interval [CI]: 0.324-6.706; Table 2). On multivariate analysis, NAFLD was not significantly associated with RFS (hazard ratio: 1.297, 95\% CI: 0.263-6.399; Table 2). Reported

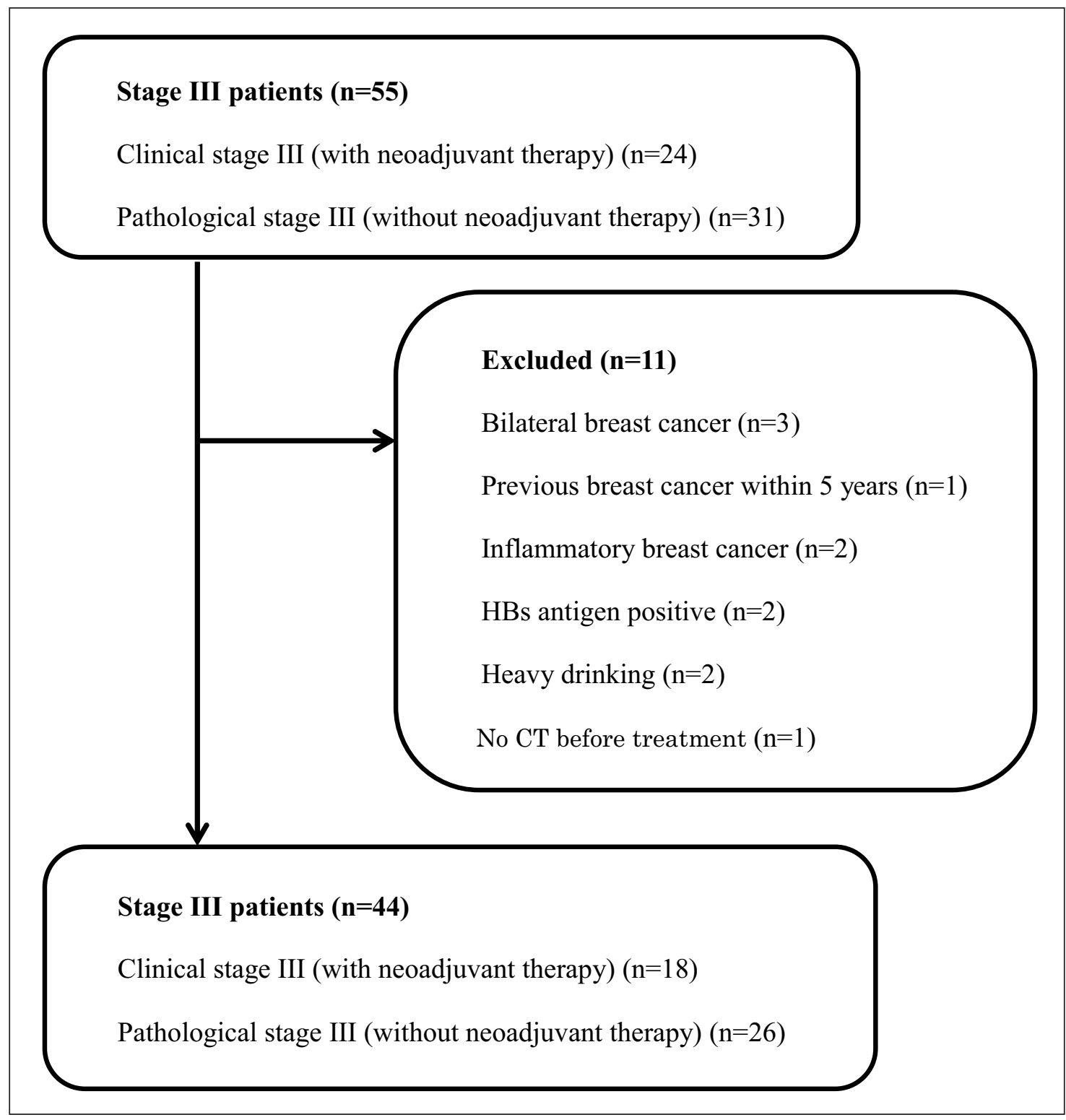

Figure 1 Flow diagram. 


\begin{tabular}{|c|c|c|c|}
\hline CHARACTERISTIC & $\begin{array}{l}\text { WITH NAFLD } \\
(\mathrm{N}=6)\end{array}$ & $\begin{array}{l}\text { WITHOUT NAFLD } \\
(\mathrm{N}=38)\end{array}$ & $\begin{array}{l}\text { ALL PATIENTS } \\
(\mathrm{N}=44)\end{array}$ \\
\hline Age (year) & $68.5[45-81]$ & 61.5 [29-90] & 63 [29-90] \\
\hline BMI $\left(\mathrm{kg} / \mathrm{m}^{2}\right)$ & $23.8[21.7-28.4]$ & $21.7[15.9-32.1]$ & $22.2[15.9-32.1]$ \\
\hline Sex Female & $6(100 \%)$ & $38(100 \%)$ & $44(100 \%)$ \\
\hline Premenopausal & $2(33 \%)$ & $11(29 \%)$ & $13(30 \%)$ \\
\hline Hypertension & $3(50 \%)$ & $13(34 \%)$ & $16(36 \%)$ \\
\hline Diabetes mellitus & $3(50 \%)$ & $7(18 \%)$ & $10(23 \%)$ \\
\hline Dyslipidemia & $1(17 \%)$ & $8(21 \%)$ & $9(20 \%)$ \\
\hline Smoking & $0(0 \%)$ & $8(22 \%)$ & $8(18 \%)$ \\
\hline CEA (ng/ml) & $2.3[1.7-3.9]$ & $3.15[0.4-109.7]$ & 3.1 [0.4-109.7] \\
\hline CA15-3 (U/ml) & 12 [10.1-14.7] & $12.8[4.5-195.4]$ & 12.5 [4.5-195.4] \\
\hline TPA (U/l) & $29.7[16.6-111.4]$ & $23.05[2.9-418.6]$ & $23.2[2.9-418.6]$ \\
\hline Histology IDC/others & $4(67 \%) / 2$ & $33(87 \%) / 5$ & $37(84 \%) / 7$ \\
\hline Histological grade $1 / 2 / 3$ & 1/4/1 (17\%) & $11 / 19 / 5(14 \%)$ & $12 / 23 / 6(14 \%)$ \\
\hline ER positive & $4(67 \%)$ & $27(71 \%)$ & 31 (70\%) \\
\hline HER2 positive & $0(0 \%)$ & $7(18 \%)$ & 7 (16\%) \\
\hline Ki 67 (\%) & $26.2[1-84.6]$ & 23.1 [2.1-90] & 23.1 [1-90] \\
\hline $\begin{array}{l}\text { Luminal/HER2/TN } \\
\text { /Luminal-HER2 }\end{array}$ & $4 / 0 / 2 / 0$ & $24 / 4 / 7 / 3$ & $28 / 4 / 9 / 3$ \\
\hline Neoadjuvant therapy & $2(33 \%)$ & $16(42 \%)$ & $18(40 \%)$ \\
\hline $\begin{array}{l}\text { Breast surgery } \\
\text { Bt/Bp/none }\end{array}$ & $4 / 2 / 0$ & 28/9/1 & $32 / 11 / 1$ \\
\hline $\begin{array}{l}\text { Axillary surgery } \\
\mathrm{Ax} / \mathrm{SN} \rightarrow \mathrm{Ax} / \mathrm{SN} / \text { none }\end{array}$ & $6 / 0 / 0 / 0$ & 27/3/7/1 & $33 / 7 / 3 / 1$ \\
\hline pCR/Tis/T1/T2/T3/T4b & $1 / 0 / 1 / 2 / 2 / 0$ & $2 / 2 / 5 / 9 / 7 / 12$ & $3 / 2 / 6 / 11 / 9 / 12$ \\
\hline $\mathrm{pNO/N1/N2/N3}$ & $0 / 1 / 2 / 3$ & $8 / 11 / 13 / 5$ & $8 / 12 / 15 / 8$ \\
\hline Tumor diameter (mm) & $29[0-80]$ & $36[0-180]$ & $34[0-180]$ \\
\hline Node metastasis & $8[2-20]$ & 3 [1-19] & $4[0-20]$ \\
\hline Radiation & $5(83 \%)$ & $29(76 \%)$ & $34(77 \%)$ \\
\hline Endocrine therapy & $4(67 \%)$ & $24(63 \%)$ & $28(64 \%)$ \\
\hline Chemotherapy & $6(100 \%)$ & 28 (74\%) & 34 (77\%) \\
\hline
\end{tabular}

Table 1 Baseline characteristics.

$\mathrm{N}(\%)$, median [range].

Abbreviations: BMI = body mass index; CEA = carcinoembryonic antigen; CA15-3 = cancer antigen 15-3; TPA = tissue polypeptide antigen; IDC = invasive ductal carcinoma; NAFLD = nonalcoholic fatty liver disease; ER = estrogen receptor; HER2 = human epidermal growth factor receptor 2; $\mathrm{TN}$ = triple negative; $\mathrm{Bt}=$ total mastectomy; $\mathrm{Bp}=$ partial mastectomy; $\mathrm{Ax}$ = axillary lymph node dissection; $\mathrm{SN}=$ sentinel node biopsy; $\mathrm{pCR}=$ pathological complete response.

prognostic factors for breast cancer-such as older age, high histological grade, and estrogen receptor negativity-were significantly associated with a shorter RFS (Table 2).

Among both patients with and those without NAFLD, we did not observe any difference in the percentage of patients who received various breast cancer treatments including radiotherapy, endocrine therapy, and chemotherapy (Table 1). Neither cardiovascular disease (CVD) nor other cancers were associated with NAFLD.

\section{DISCUSSION}

The current study is the first to investigate the association between NAFLD and LABC. The presence of NAFLD in patients with $\angle A B C$ did not influence the prognosis or comorbidity. 


\begin{tabular}{lll}
\hline VARIABLE & $\begin{array}{l}\text { UNIVARIATE ANALYSIS } \\
\text { HAZARD RATIO }(95 \% \mathrm{CI})\end{array}$ & $\begin{array}{l}\text { MULTIVARIATE ANALYSIS } \\
\text { HAZARD RATIO }(95 \% \mathrm{CI})\end{array}$ \\
\hline Age $>=50$ & $5.616(0.730-43.231)$ & \\
\hline BMI $>25$ & $0.979(0.264-3.638)$ & $0.877(0.227-3.392)$ \\
\hline NAFLD & $1.474(0.324-6.706)$ & $1.297(0.263-6.399)$ \\
\hline Diabetes mellitus & $1.600(0.488-5.242)$ & $1.547(0.444-5.393)$ \\
\hline smoking & $0.330(0.043-2.563)$ & \\
\hline Histology others & $1.175(0.256-5.388)$ & \\
\hline Histological grade 3 & $4.617(1.358-15.706)$ & \\
\hline ER negative & $5.006(1.626-15.407)$ & \\
\hline HER2 positive & $0.714(0.158-3.232)$ & \\
\hline Ki $67>14 \%$ & $3.762(0.479-29.541)$ & \\
\hline Node metastases $>=4$ & $2.449(0.782-7.668)$ & \\
\hline
\end{tabular}

Table 2 Univariate and multivariate analyses for recurrence free survival. Univariate analysis by log-rank test.

Multivariate analysis by Cox proportional hazards.

Abbreviations: BMI = body mass index; NAFLD = nonalcoholic fatty liver disease; $\mathrm{ER}$ = estrogen receptor; HER2 = human epidermal growth factor receptor 2; CI = confidence interval.

The results of the current study imply that mild NAFLD based on the criteria by Iwasaki et al. criteria [14] is not a prognostic factor for $L A B C$. This is inconsistent with the results of previous studies that showed that NAFLD was a prognostic factor for breast cancer recurrence [2, 3]. This might be because the poor prognosis for $L A B C$ might outweigh that for NAFLD. There are three major differences between previous studies and our study [2]. These three differences could act as biases to overestimate the impact of NAFLD on prognosis. First, the inclusion criteria included patients with stages 0 to III breast cancer, and stage III accounted for only $20 \%$ of the total cases. Second, pathological factors were not considered, such as the number of lymph node metastases, histological grade, the Ki-67 index, and the presence of lymphovascular invasion. Third, the definition of fatty liver on CT was strict. NAFLD was diagnosed when the attenuation of the liver was $<40$ Hounsfield units (HU) or $10 \mathrm{HU}$ lower than that of the spleen. When these criteria were applied to the patients included in the current study, none of them had NAFLD. Accordingly, whether more severe NAFLD is a prognostic factor in patients with $L A B C$ requires further study.

In the present study, all the patients who were scheduled to receive anthracycline were able to complete treatment and no patients had second cancers. Anthracycline could induce dose-related cardiomyopathy. Among the risk factors for anthracycline cardiotoxicity, hypertension, diabetes mellitus, and cardiac disease are associated with NAFLD [7, 8, 15, 16]. In addition, the leading causes of death are CVD, malignant disease, and liver-related disease [4]. Although this inference was made using data of a small cohort over a short time period, CVD and secondary cancer may not occur in patients with $L A B C$ with NAFLD. Physicians and patients with $L A B C$ should not be concerned about the presence of NAFLD while making the treatment choices.

This study has several strengths. First, the detailed pathology was included; second, two blinded, independent radiologists determined fatty liver on $\mathrm{CT}$; and third, regular follow-up was performed, including annual CT and 3-monthly visits.

However, the study has two limitations. First, this was a retrospective study with a small sample size, and no patients had severe NAFLD. This may underestimate the prognostic impact of NAFLD. Second, a few patients did not receive standard therapy because of older age, mental disease, and patient preference. This could increase the risk of relapse.

\section{CONCLUSION}

Because of the poor prognosis of locally advanced breast cancer, we should not omit breast cancer treatments, even in the presence of mild NAFLD.

Physicians and patients with $L A B C$ should not worry about the prognostic impact of mild NAFLD. Further study with more patients and other races is needed.

\section{ADDITIONAL FILE}

The additional file for this article can be found as follows:

- Supplementary Table 1. The STROCSS 2019

Guideline. DOI: https://doi.org/10.29337/ijsonco.63.s1 
ABBREVIATIONS

CI Confidence interval

CT Computed tomography

CVD Cardiovascular disease

HU Hounsfield units

LABC Locally advanced breast cancer

NAFLD Nonalcoholic fatty liver disease

PET/CT Positron emission tomography/computed tomography

RFS Recurrence-free survival

\section{COMPETING INTERESTS}

The authors have no competing interests to declare.

\section{AUTHOR AFFILIATIONS}

Tomoe Taji (iD orcid.org/ 0000-0002-1309-2763

Department of Breast Surgery, Hyogo Prefectural Amagasaki General Medical Center, Amagasaki, Japan

Yuki Kataoka iD orcid.org/0000-0001-7982-5213 Department of Clinical Research Center, Hyogo Prefectural Amagasaki General Medical Center, Amagasaki, Japan

\section{Hirofumi Suwa}

Department of Breast Surgery, Hyogo Prefectural Amagasaki General Medical Center, Amagasaki, Japan

Ai Yamaguchi iD orcid.org/0000-0001-6343-2597

Department of Breast Surgery, Hyogo Prefectural Amagasaki General Medical Center, Amagasaki, Japan

\section{Kazuna Kawabata}

Department of radiology, Hyogo Prefectural Amagasaki General Medical Center, Amagasaki, Japan

\section{Marina Shimizu}

Department of radiology, Hyogo Prefectural Amagasaki General Medical Center, Amagasaki, Japan

\section{Makoto Umeda}

Department of Gastroenterology and Hepatology, Hyogo

Prefectural Amagasaki General Medical Center, Amagasaki, Japan

\section{REFERENCES}

1. Nseir W, Abu-Rahmeh Z, Tsipis A, Mograbi J, Mahamid M. Relationship between non-alcoholic fatty liver disease and breast cancer. Isr Med Assoc J. 2017; 19(4): 242-245.

2. Lee YS, Lee HS, Chang SW, et al. Underlying nonalcoholic fatty liver disease is a significant factor for breast cancer recurrence after curative surgery. Med (United States). 2019; 98(39): e17277. DOI: $h$ ttps://doi.org/10.1097/MD.0000000000017277

3. Yan M, Wang J, Xuan Q, Dong T, He J, Zhang Q. The Relationship Between Tamoxifen-associated Nonalcoholic Fatty Liver Disease and the Prognosis of Patients With Early-stage Breast Cancer. Clin Breast Cancer. 2017; 17(3): 195-203. DOI: https://doi.org/10.1016/j.clbc.2016.12.004

4. Adams LA, Lymp JF, St. Sauver J, et al. The natural history of nonalcoholic fatty liver disease: A population-based cohort study. Gastroenterology. 2005; 129(1): 113-121. DOI: https://doi.org/10.1053/j. gastro.2005.04.014

5. Ekstedt M, Franzén LE, Mathiesen UL, et al. Long-term follow-up of patients with NAFLD and elevated liver enzymes. Hepatology. 2006; 44(4): 865-873. DOI: https:// doi.org/10.1002/hep.21327

6. Cardoso F, Kyriakides S, Ohno S, et al. Early breast cancer: ESMO Clinical Practice Guidelines for diagnosis, treatment and follow-up. Ann Oncol. 2019; 30(8): 1194-1220. DOI: https://doi.org/10.1093/annonc/mdz173

7. Henriksen PA. Anthracycline cardiotoxicity: An update on mechanisms, monitoring and prevention. Heart. 2018; 104(12): 971-977. DOI: https://doi.org/10.1136/ heartjnl-2017-312103

8. Bloom MW, Hamo CE, Cardinale D, et al. Cancer TherapyRelated Cardiac Dysfunction and Heart Failure: Part 1: Definitions, Pathophysiology, Risk Factors, and Imaging. Circ Hear Fail. 2016; 9(1): e002661. DOI: https://doi. org/10.1161/CIRCHEARTFAILURE.115.002661

9. Agha R, Abdall-Razak A, Crossley E, Dowlut N, Iosifidis C, Mathew G. STROCSS 2019 Guideline: Strengthening the reporting of cohort studies in surgery. Int J Surg. 2019; 72: 156-165. November. DOI: https://doi.org/10.1016/j. ijsu.2019.11.002

10. Brierley JD, Gospodarowicz MK, Wittekind C. TNM Classification of Malignant Tumours. John Wiley \& Sons; 2017. DOI: https://doi.org/10.1002/9780471420194. tnmc26.pub3

11. Lakhani SR. WHO Classification of Tumours of the Breast. International Agency for Research on Cancer; 2012.

12. Tryfonidis K, Senkus E, Cardoso MJ, Cardoso F. Management of locally advanced breast cancerperspectives and future directions. Nat Rev Clin Oncol. 2015; 12(3): 147-162. DOI: https://doi.org/10.1038/ nrclinonc. 2015.13

13. Gong Y, Liu YR, Ji P, Hu X, Shao ZM. Impact of molecular subtypes on metastatic breast cancer patients: A SEER population-based study. Sci Rep. 2017; 7: 1-10. February. DOI: https://doi.org/10.1038/srep45411

14. Iwasaki M, Takada Y, Hayashi M, et al. Noninvasive evaluation of graft steatosis in living donor liver transplantation. Transplantation. 2004; 78(10): 1501-1505. DOI: https://doi.org/10.1097/01. TP.0000140499.23683.0D

15. Nassir F, Scott Rector R, Hammoud GM, Ibdah JA. Pathogenesis and Prevention of Hepatic Steatosis. 2015; 11(3): 167-175

16. Lazo M, Hernaez R, Eberhardt MS, et al. Prevalence of nonalcoholic fatty liver disease in the United States: The third national health and nutrition examination survey, 1988-1994. Am J Epidemiol. 2013; 178(1): 38-45. DOI: https://doi.org/10.1093/aje/kws448 
TO CITE THIS ARTICLE:

Taji T, Kataoka Y, Suwa H, Yamaguchi A, Kawabata K, Shimizu M, Umeda M. Influence of Nonalcoholic Fatty Liver Disease on Locally Advanced Breast Cancer: A Retrospective Cohort Study. International Journal of Surgery: Oncology. 2021; 6(1), 18-24. DOI: https://doi. org/10.29337/ijsonco.63

Submitted: 30 January 2021 Accepted: 18 February 2021 Published: 23 March 2021

COPYRIGHT:

(c) 2021 The Author(s). This is an open-access article distributed under the terms of the Creative Commons Attribution 4.0 International License (CC-BY 4.0), which permits unrestricted use, distribution, and reproduction in any medium, provided the original author and source are credited. See http://creativecommons.org/licenses/by/4.0/.

IJS Oncology is a peer-reviewed open access journal published by IJS Publishing Group. 